\title{
PHOTOREFLECTANCE STUDIES OF InGaAs/GaAs/AlGaAs SINGLE QUANTUM WELL LASER STRUCTURES
}

\author{
T.J. OCHALSKI, J. ŻUK \\ Institute of Physics, M. Curie-Skłodowska University \\ Pl. M. Curie-Skłodowskiej 1, 20-031 Lublin, Poland \\ K. REgiŃSKI AND M. BUGAJSKI \\ Institute of Electron Technology, Al. Lotników 32/46, 02-668 Warsaw, Poland
}

We report on photoreflectance investigations of strained-layer $\mathrm{In}_{0.2} \mathrm{Ga}_{0.8} \mathrm{As} / \mathrm{GaAs}_{\mathrm{Al}} / \mathrm{Al}_{0.3} \mathrm{Ga}_{0.7} \mathrm{As}$ single quantum well laser structures grown by molecular beam epitaxy. All the observed photoreflectance spectral features were assigned to the $\mathrm{e}-\mathrm{hh}$ transitions with $\Delta n=0$. The transition energies were determined and compared to their values calculated within the envelope function approximation. Assuming that one third of the total strain in the central $\mathrm{In}_{0.2} \mathrm{Ga}_{0.8}$ As layer is relaxed by biaxial deformation of surrounding thin GaAs layers, it is possible to explain reasonably the results of our photoreflectance experiment.

PACS numbers: 78.66.Fd, 78.20.-e

The band structure of III-V semiconductors is considerably modified under biaxial strain (compressive or tensile), occurring in thin epitaxial layers grown on lattice mismatched substrates. The stress in the layers results in removing $k=0$ valence band degeneracy, changes in the band gap and anisotropy of hole dispersion in the valence band. The effective masses of both heavy holes (HH) and light holes ( $\mathrm{LH})$ are different for in-plane directions and for the direction perpendicular to the quantum well plane. Since the quantum well potential already removes degeneracy between the light and heavy holes, situating the heavy hole band at lower energy with respect to the bottom of the well, the inclusion of strain can either further increase or quite opposite, decrease the value of heavy hole light hole splitting, depending on whether the in-plane strain in quantum well is compressive or tensile. Ultimately it can lead to the reversal of band ordering in the valence band. Additionally, the anticrossing behavior of valence subbands has to be taken into account by any serious calculations of valence band dispersion in strained quantum wells. The above-mentioned effects are of primary importance 
when calculating any physical effect depending on the in-plane density of states in the quantum well, such as for example optical gain in the material.

The samples studied were MBE-grown strained-layer laser structures designed for the emission at $\lambda=0.98 \mu \mathrm{m}$. The active region of the structure consisting of the undoped $\operatorname{In}_{0.2} \mathrm{Ga}_{0.8}$ As single quantum well of the thickness $L=$ $100 \AA$, embedded in $50 \AA$ thick GaAs layers and was surrounded by $0.1 \mu \mathrm{m}$ thick $\mathrm{Al}_{0.3} \mathrm{Ga}_{0.7} \mathrm{As}$ layers. The structures were grown on a Riber $32 \mathrm{P}$ solid source machine. Growth conditions, i.e., the substrate temperature and appropriate molecular fluxes were optimized in a series of test growth runs.

In order to gain more information about these structures, in particular to determine precisely all transition energies for the quantum well, the photoreflectance (PR) investigations have been carried out. PR spectra were taken at $300 \mathrm{~K}$ in a standard experimental setup [1] with a spectral resolution of $4 \mathrm{meV}$. To minimize sample's heating which can lead to uncontrolled shifts of PR peaks, a $1 \mathrm{~mW} \mathrm{Ar}{ }^{+}$laser unfocused beam was used in this study. In addition, PR measurements in the temperature range from 77 to $450 \mathrm{~K}$ were carried out to exclude possible interference or GaAs substrate effects on the PR spectra.

The lattice constant of GaAs is equal to $5.6533 \AA$, whereas that of InAs equals $6.0584 \AA$, which results in a. $7 \%$ lattice mismatch between those two semiconductors [2]. The consequence of the above is that conduction and valence bands of strained layer lattice mismatched $\mathrm{In}_{x} \mathrm{Ga}_{1-x}$ As system are altered by the presence of biaxial strain. The room temperature band gap of $\operatorname{In}_{x} \mathrm{Ga}_{1-x} \mathrm{As}$ bulk unstrained ternary alloy varies from $1.424 \mathrm{eV}(\mathrm{GaAs})$ to $0.36 \mathrm{eV}$ (InAs), i.e., $E_{\mathrm{g}}(x)=1.424-1.619 x+0.555 x^{2}[2]$.

The biaxial deformation of the elastically deformed layer contains both the hydrostatic and uniaxial components. The first one results in a rigid band shift, the second in splitting of $\mathrm{HH}$ and $\mathrm{LH}$ bands, each being pushed in the opposite direction from the center. The strained band gaps are obtained under the assumption that even at $k=0$, in bulk material, degeneracy between the $\mathrm{HH}$ and the $\mathrm{LH}$ bands is removed.

The $\mathrm{In}_{x} \mathrm{Ga}_{1-x}$ As layer grown on GaAs is always compressed, therefore the $\mathrm{HH}$ band is located above the LH band. Although the lattice mismatched, the relaxed layer is an energetically favorable state, because the elastically deformed layer with a thickness less than the critical thickness is still in a local energy minimum state due to the existence of energy barrier for the dislocation generation process.

The conduction (C) to $\mathrm{HH}$ energy gap $E_{\mathrm{g}}^{\mathrm{C}, \mathrm{HH}}$ composition dependence for compressively strained $\mathrm{In}_{x} \mathrm{Ga}_{1-x}$ As grown on GaAs is given by the following relation [2]: $E_{\mathrm{g}}^{\mathrm{C}, \mathrm{HH}}=1.424-1.06 x+0.08 x^{2}[\mathrm{eV}]$. The splitting energy $(S)$ between light hole and heavy hole bands also depends on the composition: $S=E_{\mathrm{g}}^{\mathrm{C}, \mathrm{LH}}-E_{\mathrm{g}}^{\mathrm{C}, \mathrm{HH}}=$ $0.465 x-0.33 x^{2}[e V][2]$. As a result, the potential barrier for the light hole band quantum well is smaller than the one for the heavy hole band and decreases with increasing indium content in the alloy.

Figure 1 shows the PR spectrum of the investigated strained-layer laser structure. The performed least-squares fit was based on the low-field third-derivative 


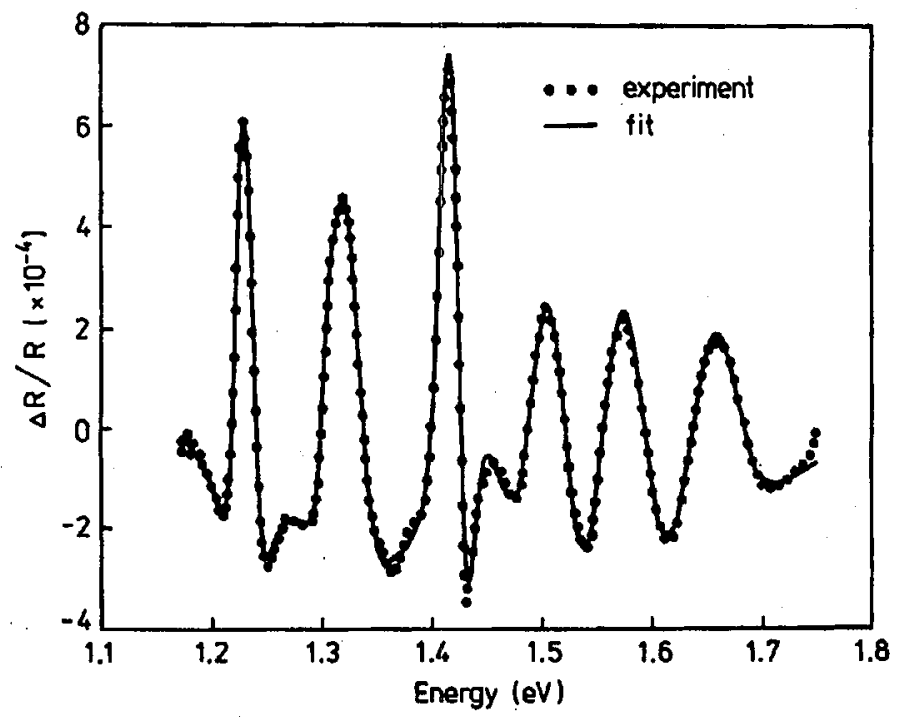

Fig. 1. Photoreflectance spectrum (dotted line) of $\operatorname{In}_{0.2} \mathrm{Ga}_{0.8} \mathrm{As} / \mathrm{GaAs} / \mathrm{Al}_{0.3} \mathrm{Ga}_{0.7} \mathrm{As}$ single quantum well laser structure at $300 \mathrm{~K}$. The solid line is a TDFF fit as discussed in the text.

\section{TABLE}

Quantum well transition energies (in eV) calculated and experimentally determined from $P R$ measurements.

\begin{tabular}{c|c|c|c}
\hline \hline Transition & Experiment & Theory (1) & Theory (2) \\
\hline le-1hh & 1.229 & 1.2507 & 1.2307 \\
1e-1lh & 1.304 & 1.3329 & 1.2917 \\
2e-2hh & 1.320 & 1.3486 & 1.3286 \\
2e-2lh & 1.422 & 1.4380 & 1.4082 \\
3e-3hh & 1.437 & 1.4638 & 1.4444 \\
3e-3lh & & 1.5626 & 1.5371
\end{tabular}

functional form (TDFF) for line shapes, proposed by Aspnes [3]. Two-dimensional density of states was used in the calculations. The fit is quite satisfactory in the investigated energy range. The observed PR spectral features were assigned to e-hh transitions with $\Delta n=0$ in the quantum well structure, and are listed in Table. The $\Delta n=2$ transitions are from 2 to 3 orders of magnitude lower in intensity and were neglected in the analysis. A relatively small width of the strong PR feature at $1.229 \mathrm{eV}$, as well as that corresponding to a weaker line at $1.304 \mathrm{eV}$ reflect a good localization of the deeper lying states in the quantum well. Also the PR feature positioned at $1.422 \mathrm{eV}$, i.e., close to the $E_{\mathrm{g}}$ value for $\mathrm{GaAs}$, is quite narrow.

For the calculation of electron and hole confined energy levels it has been assumed that band gap discontinuity between the central. $\operatorname{In}_{x} \mathrm{Gra}_{1-x} \mathrm{As}$ layer and 
the surrounding GaAs barriers is partitioned as $\Delta E_{\mathrm{c}}: \Delta E_{\mathrm{v}}=0.6: 0.4$. This is indeed: $\Delta E_{\mathrm{c}}: \Delta E_{\mathrm{HH}}=0.6: 0.4$ correct for the case in question, whereas the barrier height for light holes results from the specific value of LH and HH bands splitting $S$ :

The hole effective masses in the $z$-direction, i.e., perpendicular to the quantum well have been calculated from Luttinger parameters, for which values for a given $\operatorname{In}_{x} \mathrm{Ga}_{1-x}$ As composition were obtained by linear interpolation between the known values for GaAs and InAs [2]. For $x=0.2$ the following values have been obtained: $\gamma_{1}=9.414$ and $\gamma_{2}=3.354$. The appropriate hole masses are: $m_{\mathrm{h} z}=1 /\left(\gamma_{1}-2 \gamma_{2}\right)=0.370 m_{0}$ and $m_{\mathrm{l} z}=1 /\left(\gamma_{1}+2 \gamma_{2}\right)=0.062 m_{0}$. The electron effective mass is isotropic and has the following composition dependence: $m_{\mathrm{c}}(x)=0.067-0.04 x[2]$.

The confined energy levels for electrons and holes have been calculated within the envelope function approximation [4]. The Schrödinger equation for the envelope function has been solved numerically by using a finite difference method. The results of calculations are summarized in Table which lists the optical transition energies between the quantized levels with the principal quantum number $n$ up to 3 . The exciton effects have been neglected for the first approximation.

The column marked Theory (1) shows the results of calculations assuming that the whole mismatch strain is accommodated by the central $\mathrm{In}_{0.2} \mathrm{Ga}_{0.8}$ As layer. The calculated energies are, however, consistently higher than the experimental results. To account for the observed discrepancy we postulate that a part of the compressive strain in the $\mathrm{In}_{0.2} \mathrm{Ga}_{0.8}$ As layer is relieved by the biaxial deformation of the thin GaAs layers situated in between the $\mathrm{In}_{0.2} \mathrm{Ga}_{0.8} \mathrm{As}$ and $\mathrm{Al}_{0.3} \mathrm{Ga}_{0.7} \mathrm{As}$

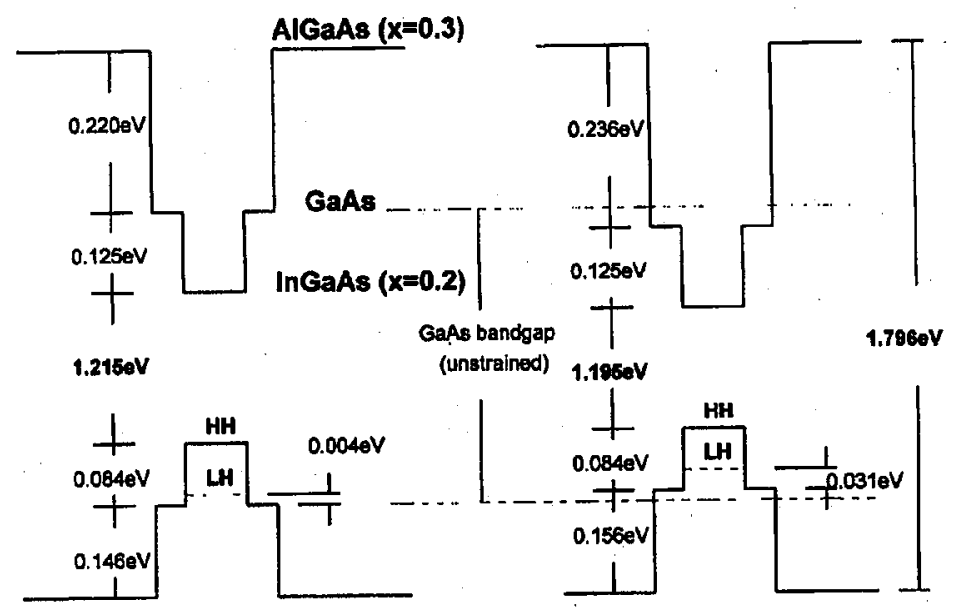

Fig. 2. Band structure of $\operatorname{In}_{0.2} \mathrm{Ga}_{0.8} \mathrm{As} / \mathrm{GaAs}_{\mathrm{s}} / \mathrm{Al}_{0.3} \mathrm{Ga}_{0.7}$ As single quantum well calculated under the following assumptions: (1) that all strain is accommodated by the central $100 \AA$ thick $\operatorname{In}_{0.2} \mathrm{Ga}_{0.8}$ As layer (left part) and, (2) that one third of the total strain is relaxed by the deformation of the $50 \AA$ thick GaAs layers (right part). 
layers. This will result in tensile strained GaAs with a consequently modified band structure shown in Fig. 2. The transition energies of the quantum well heterostructure, calculated under the assumption that one third of the total strain energy of deformation is relaxed by the compliant deformation of GaAs layers, are listed in the column labeled Theory (2) of Table. This time the agreement between calculations and experiment is much better.

\section{References}

[1] T.J. Ochalski, J. Żuk, L.A. Vlasukova, Acta Phys. Pol. A 92, 935 (1997).

[2] P.S. Zorry, Jr., Quantum Well Lasers, Academic Press, Boston 1993, p. 79.

[3] D.E. Aspnes, Surf. Sci. 37, 418 (1973).

[4] G. Bastard, Wave Mechanics Applied to Semiconductor Heterostructures, Les Editions de Physique, Paris 1994. 